Helga Krïger-Kirn

\title{
Somatisches Wissen artikulieren. Annäherungen an die leiblichen Erfahrungen von Schwangerschaft und von Leihmutterschaft
}

Seit einigen Jahren findet der Themenkomplex Schwangerschaft und Mutterschaft in der Geschlechterforschung und in der Soziologie verstärkte Aufmerksamkeit (z. B. Schadler, 2013; Hirschauer et al. 2014; Niekrenz 2017; Heitzmann 2017). Dabei ist die Dekonstruktion von Geschlechterstereotypien wie auch die Anerkennung vielfältiger Formen von Mutterschaft (z. B. Tolasch/Seehaus 2016) von Bedeutung sowie praxeologische Perspektiven, die Schwangerschaft als einen "mehrstufige[n] Evidenzierungsprozess" (Hirschauer et al. 2014, 259) untersuchen. Im Unterschied dazu nimmt der vorliegende Beitrag die körperbasierten Erfahrungen von Schwangerschaft aus einer sozialpsychologischen Perspektive in den Blick und fragt danach, wie sich der werdenden Mutter im Prozess der Schwangerschaft Diskurse als Ebene des Gesellschaftlichen vermitteln und wie sie subjektiv angeeignet werden. Orientiert an einem psychoanalytischen Subjekt-Verständnis und mit Fokus auf die körperlichen und leiblichen Erfahrungen von schwangeren Frauen ${ }^{\star 1}$ soll untersucht werden, welche gesellschaftlichen Wissensbestände in welcher Weise für Schwangere relevant werden und auch (unbewusste) Ideale von Mutterschaft vertiefen. Der Begriff der Erfahrung dient dabei sowohl als Bezeichnung für das subjektive Erleben als auch als zentrale Analysekategorie und rückt den Körper als Referenzpunkt im Schnittfeld eigenleiblicher Erfahrungen und sozialer geschlechtlicher Codierungen ins Zentrum. Erkenntnistheoretisch wird so an das zentrale Anliegen feministischer Forschung angeknüpft, die subjektiven Erfahrungen der Frauen zum Ausgangspunkt wissenschaftlicher Erkenntnisse und Forschungskonzepte zu machen.

Der vorliegende Beitrag gliedert sich in drei Abschnitte. Zunächst wird in Auseinandersetzung mit der einschlägigen körpersoziologischen und

1 Diese Bezeichnung schließt auch transsexuelle und genderqueere Menschen ein, die ebenfalls Erfahrungen mit Schwangerschaft haben. Ich formuliere meine Überlegungen mit Bezug auf die gegenwärtige symbolische Ordnung der Zweigeschlechtlichkeit (Rede von Männern und Frauen). Als Hinweis auf den Konstruktionscharakter

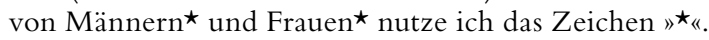


kulturwissenschaftlichen Forschung gezeigt, dass die Erlebnisweisen von Schwangerschaft und die sie begleitenden leiblichen Erfahrungen eine Leerstelle im Forschungsfeld Schwangerschaft darstellen. Jenseits von phänomenologischer Beschreibung gibt es nur selten empirisch fundierte Artikulationen von Schwangeren` selbst. Die Position der Schwangeren` als Subjekt mit eigener Wahrnehmung ihres sich verändernden Körpers und eigener Stimme kommt in der wissenschaftlichen Reflexion kaum vor (I). Verbunden damit ist auch die methodisch-methodologische Frage, wie leibliche Erfahrung und leibliches Erleben in seiner meist unvollständig artikulierbaren Form empirisch erfasst werden kann. Zur Annäherung an die Dimension der leiblichen Erfahrung von Schwangerschaft werden im Anschluss verschiedene Beispiele leiblicher und psychischer Artikulationen des Erlebens von Schwangerschaft und des Schwanger-Seins vorgestellt und diskutiert (II) und im dritten Abschnitt mit Fokus auf die spezifischen Schwangerschaftserfahrungen von Leihmüttern kontrastiert (III).

\section{I: Schwangerschaft als Gegenstand sozialwissenschaftlicher Forschung}

Im Kontext von Körpersoziologie und Kulturwissenschaften hat die empirische Erforschung verschiedener Aspekte von Mutterschaft und Schwangerschaft zugenommen (Lange / Ullrich 2018; Niekrenz 2017; Makowsky / Schücking 2013). Untersucht werden geschlechterstereotypisierende $\mathrm{Zu}-$ schreibungen und elterliche Zuständigkeiten sowie die Frage, wie werdende Elternschaft praktisch vollzogen und erzeugt wird. Mit Fokus auf diskursiv-performative Aktionen werden die Zusammenhänge von Wissensbeständen, Artefakten und Tätigkeiten untersucht, welche die Evidenz herstellen, "schwanger zu sein und Mutter/Eltern zu werden" (Schadler 2013, 4; auch: Hirschauer et al. 2014; Kortendiek et al. 2017; Heitzmann 2017; Malich 2017).

Auch Forschungen, die sich einer kritischen Diskussion der reproduktiven Medizin sowie den Möglichkeiten und Bedingungen guter gesundheitlicher Versorgung widmen, rücken die (werdende) Mutter in den Blick. Sie zeigen, dass mit dem Einzug des medizinischen Risikokonzepts in die Schwangerenvorsorge und Geburtshilfe die Reduktion von Schwangerschaft auf einen somatischen Zustand und auf einen Gegenstand medizintechnischer Intervention verbunden war (Duden 1987) und zu einer zunehmenden Instrumentalisierung der Schwangeren und ihres Körpers führte. Durch die Ausbreitung von Visualisierungstechniken, wie 
z.B. Ultraschall, wird der Fötus als Kind mit eigenem Körper im Körper der Schwangeren figuriert und stützt die Vorstellungen einer Trennung des Körpers der Frau vom Körper des ungeborenen Kindes. Der Embryo erscheint als Person mit eigenen Bedürfnissen und Rechten und tritt zugleich als Bedingung der Funktionalisierung des Mutterkörpers in Erscheinung (Achtelik 2015; Kneuper 2005).

Sowohl die Personalisierung des Embryos als auch die Trennung von schwangerem Körper und Embryo überlagern die Subjektivität der Schwangeren ${ }^{\star}$ mit ihren eigenen Rechten und Erfahrungen. Feministische Analysen zeigen, dass es im Zuge der neuen Technologien der Reproduktionsmedizin und der Schwangerschaftsüberwachung zu einer Verschiebung von ehemals disziplinierenden zu nunmehr selbstfürsorglichen Formen der Fremdbestimmung gekommen ist (Heimerl 2013; Ullrich 2012). So fand die US-amerikanische Medizinethnologin Susan Erikson (2012) heraus, dass Schwangere weder an der Verlässlichkeit der Technik noch am Können der Ärzt ${ }^{\star}$ innen, sondern vornehmlich an sich selbst zweifeln. Barbara Duden (2002) beschrieb, dass sich das Körpererleben Schwangerer durch neue medizinische Techniken gegenüber einer früheren, vornehmlich haptischen Selbstwahrnehmung derartig verändert habe, dass von einem "epistemischen Bruch in der Körperwahrnehmung" gesprochen werden könne. Demgegenüber legen neuere Studien zur Nutzung medizinischer Technologien in der Schwangerschaft nahe, dass körperbezogene Wahrnehmungsformen wie Tasten und Spüren nicht verschwunden sind, sich aber durch den Einzug von Technik verändert haben (Sänger et al. 2013).

Des Weiteren gibt es Forschungen, die Prozesse der Veränderung von Identität, Subjektivität und Biografie werdender Mütter als Übergangspassage oder körpervermittelte Statuspassage untersuchen (Niekrenz 2017; Mozygemba 2011). So zeigt Kati Mozygemba auf der Grundlage einer qualitativen Interviewstudie, wie Frauen den Statusübergang in das Mutter-Werden mittels der Verschränkung von Körperwissen und Leiberleben realisieren. Sie arbeitet heraus, dass die Frauen »vor der Geburt Mutter werden« (Mozygemba 2011, 143): Die Vergegenständlichung der Schwangerschaft (Körperwissen) verbinde sich mit dem Spüren der Schwangerschaft (Leiberleben) und konkretisiere für die Frauen ihr subjektives Schwanger-Sein und Mutter-Werden (ebd., 144). Auch aus der Psychoanalyse, bei der das körperliche und psychische Erleben von Subjekten im Mittelpunkt steht, gibt es vereinzelt Studien zum Thema Schwangerschaft (z.B. Balsam 2012; Stephanos-Auhagen 2017). Insgesamt aber bleibt auch in der psychoanalytischen Wissenschaft unterbelichtet, wie die 
körperbasierten leiblichen Erfahrungen einer Schwangerschaft von Müttern / Frauen ${ }^{\star}$ psychisch verarbeitet werden und das Körper-Selbst-Verhältnis verändern (Krüger-Kirn 2015a; 2017).

Es zeigt sich also, dass das konkrete Erleben von Schwangerschaft und die leiblichen Wahrnehmungen, Empfindungen und Deutungen dieser spezifischen Körperlichkeit durch die Schwangeren ${ }^{\star}$ auch in der sozialund kulturwissenschaftlichen Forschung zum Thema Schwangerschaft selten thematisiert und untersucht werden. Damit aber bleiben Expert ${ }^{\star}$ innen-Wissen, mögliche Artikulationen nicht-instrumenteller und nichtfunktionalistischer Mutterschaft im Dunkeln und Möglichkeitsräume weiblicher Subjektivität verschlossen.

\section{II.1 Methodologische Annäherungen: Schwangerschaft als leibliche Erfahrung erfassen}

Um diese Leerstelle zu füllen, schlage ich im Folgenden eine psychoanalytische Perspektive auf die Leib-Erfahrungen schwangerer Frauen ${ }^{\star}$ vor, in der die Trias von Körper, Diskurs und Psyche/Subjekt verknüpft und das (Spannungs-)Verhältnis von organisch-materiellen und somato-psychischen Verkörperungen als wechselseitige Abhängigkeit von Körper und Diskurs auf der Ebene leiblich spürbarer Körpererfahrungen betrachtet wird. Im Rückgriff auf das Konzept der psychischen Mentalisierungs- und Symbolisierungsfähigkeit (Fonagy / Target 2003), das die subjektiven Überzeugungen und Handlungsweisen als eingebettet in einen Interaktionsprozess mit bedeutenden Anderen konzeptualisiert, soll deutlich werden, wie die subjektiven Körperwahrnehmungen auf komplexe Weise mit den herrschenden geschlechtlichen und wissenschaftlich-medizinischen Diskursen in Bezug stehen und somit keinen biologischen, bloß somatischen Referenzpunkt darstellen. Dies legt für das Verstehen von Schwangerschaft nahe, sie nicht als Zustand, sondern als einen Prozess zu fassen, der durch die fortschreitenden körperlichen Veränderungen psychisch immer wieder neu angeeignet werden muss.

Die Frage, wie man sich diesen Sinngebungsprozess der Subjektivierung im Kontext der Körpererfahrungen von Schwangerschaft vorstellen kann, lenkt den Blick auf somatisch-leibliche Interaktionen. Die unumstößliche körperliche Präsenz einer Schwangerschaft konfrontiert Frau^ in spezifischer Weise mit ihrem Körper und ihren Körpergrenzen. Die erlebten Veränderungen führen dazu, dass aus dem Subjekt Frau^ mit einem bisher stabil erlebten Körper-Selbst-Verhältnis in der Schwangerschaft ein Indi- 
viduum mit nicht planbaren Körpergrenzen wird (Krüger-Kirn 2015b). Mit der Veränderung der Körpergrenzen gehen Verunsicherungen einher, die auf der psychischen Ebene eine Integration in das bisherige Körper-Selbst-Konzept erfordern. Während der veränderte Körperzustand in den ersten drei bis vier Monaten nur für das schwangere Subjekt selbst (und ausgewählte Bezugspersonen) Bezugspunkt körperlicher und psychischer Auseinandersetzungen ist, gehen mit der Sichtbarkeit des wachsenden Bauchs die körperlichen und psychischen Transformationsprozesse zunehmend auch in einen öffentlichen Raum über. Dabei steht die Artikulation der neuen, veränderten Leiblichkeit in einem engen Zusammenhang mit der Wirkmächtigkeit kultureller Symbolisierungen von Schwangerschaft.

In der phänomenologischen Tradition wird leibliche Erfahrung als genuine Erkenntnisweise des Leibes vorgestellt. Der Bezug auf das eigenleibliche Spüren als subjektive Dimension des Somatischen und damit untrennbares Wechselverhältnis zwischen Körper und Leib spricht dem Körper als Erkenntnisinstrument eine eigenleibliche Kompetenz und Autorität zu (Gahlings 2006). Anke Abraham unterscheidet mit Bezug auf Helmuth Plessner drei Dimensionen des Körpererlebens: Erstens ein Erleben vom Körper (ich nehme etwas an meinem oder in meinem Körper wahr), zweitens ein Erleben mittels des Körpers (der Körper als Medium des Erlebens) und drittens ein Erleben des Körpers in dem fundamentalen Sinne, dass der Körper als eine eigenständige Empfindungseinheit etwas merken kann (Abraham 2010). Das leibliche Spüren bezieht sich also sowohl auf ein körperliches Außen wie Innen. In der Schwangerschaft erhält das Berührtwerden von Innen eine neue Dimension. Gegenüber dem Spüren des eigenen Inneren, das mit Schmitz (1985) als Spüren von »Leibinseln« differenziert werden kann, bezieht sich das Spüren im Prozess der Schwangerschaft sowohl auf den schwangeren Körper als auch auf das Infans. Dabei sind die Materialität des mütterlichen Körpers wie des fetalen Körpers nicht eindeutig voneinander $\mathrm{zu}$ trennen, dennoch werden beide "Körper von Gewicht" (Butler).

Dieser Prozess des Bedeutsam-Werdens der körperlichen Veränderung im Zuge einer Schwangerschaft wird leiblich-subjektiv erfahren und ist zugleich kulturell bestimmt. Weil diese Leiblichkeit auch immer unbewusste soziale und geschlechtliche Vorstellungen repräsentiert, halte ich es über ein phänomenologisches Verständnis hinausgehend für erforderlich, den Leib nicht nur als eine Verschränkung von einem von Wissen und Symbolstrukturen durchzogenen Körper und Leib zu fassen, der nur über den gemeinsamen Sprachkontext - also eine doppelte Kontingenz - zugänglich werden kann (Jäger 2004), sondern in den leiblichen Erfahrungen auch 
Dimensionen des Unbewussten anzunehmen. Eine Differenzierung des Leibverständnisses in bewusste und unbewusste Artikulationen erweitert demzufolge den epistemologischen Raum um solche Erfahrungen, die als wortlose Unsagbarkeiten im Körper gespeichert, aus dem bewussten leiblichen Erfahrungsspektrum jedoch ausgeschlossen sind und nur kryptisch bzw. verschlüsselt versprachlicht werden können (Krüger-Kirn 2018).

\section{2 Empirische Annäherungen - Vielfalt der Artikulationen}

Die methodologischen Überlegungen sensibilisieren die empirische Erfassung leiblicher Erfahrungen von Schwangerschaft dahingehend, dass sie die Aufmerksamkeit auch auf solche Artikulationen lenken, die weniger eindeutig, stärker ambivalent und vielleicht sogar unverständlich sind. Hier - und nicht in medial präsentierten Darstellungen und Erzählungen - werden Dimensionen leiblicher Erfahrung von Schwangerschaft zugänglich, die das gängige Verständnis von Schwangerschaft und Mutter-Werden zu erweitern vermögen. Dementsprechend stellt die folgende Darstellung den Versuch einer verstehenden Annäherung dar. Sie ist methodisch unsystematisch und zieht empirisches Material aus ganz unterschiedlichen Kontexten heran, um bislang Ausgeblendetes, Nicht-Gesagtes und Schwer-Verständliches einer Deutung zugänglich zu machen. Kaleidoskop- artig werden Blogbeiträge, Literatur, psychoanalytische Fallvignetten (Krüger-Kirn 2015a) sowie Passagen aus Interviews mit (werdenden) Müttern aus einer bislang unveröffentlichten Studie von Helga Krüger-Kirn und Leila Tichy zusammengestellt und kommentiert, um die Vielfalt und Bandbreite der Artikulationen leiblicher Erfahrung von Schwangerschaft zu veranschaulichen.

\section{Entgrenzung und Entfremdung}

Die körperlichen Veränderungen in der Schwangerschaft sind ein Anlass für Verunsicherungen, die leiblich erlebt und psychisch bearbeitet werden. Die Bedeutung der körperlichen Veränderung zeigt sich beispielsweise in der Fallvignette der psychoanalytischen Behandlung von Frau N., die eine Schwangerschaft plant und im Vorfeld träumt:

Frau N.: "Irgendwer hat versucht, meine Grenze zu überschreiten, hat versucht, immer wieder etwas zu machen, was unverschämt war, mir etwas zu stehlen oder so ...". 
Hier artikulieren sich Ängste vor den erwarteten Körperveränderungen in konkretistischen Vorstellungen von Angst erregenden Überschreitungen und in Form eines möglichen Verlusts.

Während sich die Angst vor den (Körper-)Veränderungen bei Frau N. auf einer imaginären Ebene abspielt, berichtet Johana Gomez in ihrem Blog über ihre Schwangerschaft und beschreibt eine leiblich erfahrene Entfremdung vom eigenen Körper:

"Mein Leib fing an, sich zu verändern. Plötzlich hatte ich wahnsinnig große Brüste und musste mich ständig ausruhen. Ich war nicht mehr ich, mein Körper wurde verfremdet und ich war der Zweck des Mutterseins" (Gomez 2017).

Die reproduktiven Körperveränderungen drängen sich in ihrer leiblichen Präsenz in das subjektive Empfinden und werden als unkontrollierbar erlebt. Gefühle der körperlichen Selbstentfremdung erschüttern in dieser Hinsicht das bisherige Körper-Selbst-Verhältnis und Frau^ fühlt sich nicht mehr als Herr` im eigenen Haus.

Gleichwohl artikuliert das beschriebene Erleben in den zwei Beispielen nicht nur individuelle psychische Verarbeitungsweisen. Sie repräsentieren zugleich Signifizierungsprozesse und geben Aufschluss darüber, wie die Körpersensationen und -veränderungen in den Kontext eines Körperwissens über Schwangerschaft gestellt werden. Dieser Signifikationsprozess geschieht im Horizont des aktuellen medizinischen Wissens darüber, was sich wann in einer Schwangerschaft ereignet und erwartungsgemäß zu verspüren ist. Dass die Körperveränderungen nicht nur als Forderung des Körpers erlebt werden, sondern mit Mutterschaftsnormen in Bezug gesetzt werden, drängt sich als Deutung auf, wenn Frau Gomez vom "Zweck des Mutterseins" spricht. Es kann vermutet werden, dass hier tradierte soziokulturelle Mutterbilder ihre Wirkung entfalten und den schwangeren Körper zum Zweck des Mutterseins funktionalisieren. Mittels dieser Bezugnahme auf stereotype Zuschreibungen wird der Körperzustand von Schwangerschaft unbewusst auf ein passives mütterliches Behältnis reduziert. Hier fehlen, so könnte man mit Barbara Duden und ihren Arbeiten zum Verlust des sinnlichen Körperwissens in der Geschichte der Körpererfahrungen in der Schwangerschaft einwenden, differenzierte Bezüge auf soziokulturelle und geschlechterspezifische Vorstellungen als notwendige Voraussetzung, um Schwangerschaftserfahrungen nicht funktionalistisch zu naturalisieren (Duden 1987). 


\section{Begehren und Potenz}

Schwangerschaft wird nicht nur gefürchtet, sondern auch gewünscht und begehrt. Irritierend ist ein Begehren nach einem Kind, das sich leiblich aufdrängt und als sehr kraftvoll erlebt wird. Eine solche Sprachlosigkeit in Bezug auf ihren Kinderwunsch thematisiert Maggie Nelson in ihrem Buch Die Argonauten«:

"Wann immer mich jemand fragte, warum ich ein Baby wollte, hatte ich keine Antwort. Aber die Stummheit des Wunsches war umgekehrt proportional zu seiner Größe» (Nelson 2015, 44).

Während Nelson versucht, ihr Begehren nach einem Kind zu verstehen, zeigt das Protokoll der Therapierstunde von Frau Ch. eine Artikulation, die einen eher narzisstisch gefärbten Selbstaspekt in den Vordergrund rückt:

Frau Ch.: "Schwanger sein und ein Baby zu haben, macht mich als Frau $z u$ etwas Besonderem, ich wäre etwas wert, würde mich von anderen Frauen unterscheiden".

Anders als bei Maggie Nelson, die zum Ausdruck bringt, dass sich ihr der Kinderwunsch leiblich aufdrängt und ihr subjektives Erleben dominiert, imaginiert Frau Ch. den schwangeren Körper als einen begehrenswerten Zustand, als eine Sehnsucht, die Frau^ gleichzeitig "zu etwas Besonderem" macht. Diese phantasierte Aufwertung vollzieht sich im imaginären Vergleich mit anderen Frauen, um sich »von anderen Frauen [zu] unterscheiden". Vordergründig drängt sich hier ein weiblicher Neidkomplex als Deutungsmuster auf, der seit Freud mit dem "Penisneid" assoziiert wird. Zugleich ist in dem Zitat aber auch der Außenblick eines etablierten (männlich patriarchalen) Blicks gegenwärtig, der ein Klischee der Weiblichkeit artikuliert, in dem `Frau sein und `Schwanger sein sammengehören und den Wert von Frauen ${ }^{\star}$ bestimmen. Im Lichte dieser affirmativen Zuschreibungen bleibt es schwierig in dem Verlangen, "sich $\mathrm{zu}$ etwas Besonderem zu machen" auch eine Sehnsucht, "schwanger zu sein« zu lesen und mit Körperpotenz zu assoziieren.

Bisher liegen keine empirischen Untersuchungen zu einem Schwangerschaftsbegehren vor, die den Kinderwunsch erklären. Auch gibt es nur wenige Beschreibungen bezüglich eines leiblich spürbaren Begehrens, schwanger zu werden. Gleichwohl kommen in solchen Beschreibungen Erfahrungen zur Sprache, die als Begehren und ein Spüren am eigenen Leib relevant werden (Krüger-Kirn 2015; Gahlings 2006). Sie geben an- 
gesichts normativer Schwangerschaftsvorstellungen Hinweise auf Begehrensformen, die hinter den diskursiven Grenzen heteronormativer Vorstellungen von Schwangerschaft liegen und für die keine Sprache vorgesehen ist (Krüger-Kirn 2017). Psychoanalytisch kann hier mit Jagenow / Mittag (1984) an ein Fruchtbarkeitsbegehren gedacht werden, das dem sexuellen Körper eine autonome Antriebskraft zuschreibt und der Wirkmacht soziokultureller Erwartungen gegenübersteht.

\section{Erleben leiblicher Intersubjektivität}

Mit fortschreitender Schwangerschaft wird der Fötus als ein vom eigenen Körper abgegrenztes, eigenständiges Wesen wahrgenommen. Die zunehmend fühlbare Vergegenständlichung des Fötus verschränkt sich mit dem leibhaftigen Spüren der Schwangerschaft. Damit konkretisiert sich für die Frau^ ihr Schwanger-Sein noch einmal anders. Wenn »ein Baby im Mutterleib ganz wörtlich Raum schafft, wo zuvor kein Raum war", wird der Körper ein Ort für ein lebendiges Wesen (Nelson 2017, 134). Diese Erfahrung der Schwangerschaft wird in dem Stundenprotokoll der psychoanalytischen Therapie von Frau O. folgendermaßen zitiert: "Ich fühle mein Baby in mir als etwas Hartes, Abgegrenztes - und gebe ihm seinen Arbeitstitel >Murmelchen In mir wächst ein Körper, der ganz anders ist als meiner und doch auch meiner ist". Diese besondere Wahrnehmung der Getrenntheit hat Iris Young (1990) als einen Prozess beschrieben, in dem das Innere des eigenen, subjektiv erlebten Körpers zur flexiblen Grenze für ein anderes Subjekt wird. Dieser neue, auf den Organismus der Schwangeren bezogene Sachverhalt verändert die leiblich fühlbaren Dimensionen, deren psychische Aneignung eine besondere Herausforderung darstellt. Denn es handelt sich um eine "radikale Intimität, radikale Differenz. Und beide sind im Körper zuhause" (Nelson 2017, 114). Indem Nelson (2017) diesen Zustand als wradikale Intimität " beschreibt, die beide Körper einschließt und doch voneinander trennt, verweist sie auf die Entstehung einer leiblich fühlbaren Beziehung von Körper zu Körper. Zudem bringt sie zum Ausdruck, dass sich zwischen beiden Körpern auch ein Erfahrungsraum aufspannt, der durch Berührtwerden von Innen entsteht und zur Basis einer außergewöhnlichen Kommunikation wird. Auf diesen Raum richtet die Schwangere ihre Phantasien, Wünsche und Ängste. Sie differenziert zwischen sich und dem Infans und entwickelt Bilder, die sie mit ihren Phantasien und Vorstellungen in Bezug setzt. Dergestalt bedeutet sie auch die fötalen Körperbewegungen und macht auf diese Weise mit ihren emotionalen und geschlechtlichen Zuweisungen aus dem fötalen Körper ein fötales Subjekt. Um zu unter- 
streichen, dass es sich bei dieser besonderen Form der leiblich fühlbaren Kommunikation nicht um eine Kommunikation zwischen zwei Körpern handelt, sondern dem Infans von der Mutter^ eine Leiblichkeit zugesprochen wird, wird hier der Begriff Zwischenleiblichkeit vorgeschlagen. Zwischenleiblichkeit in der Schwangerschaft rückt eine Position ins Zentrum, die das Spüren von Innen mit einer Bereitschaft in Bezug setzt, sich auf Unbekanntes und Fremdes einzulassen (Ettinger 2006).

Dieses Empfinden kann aber auch als befremdlich und irritierend erlebt werden, wie Johana Gomez (2017) in ihrem Blog beschreibt:

"Ich fing an, in diesem Zeitraum mit dem neuen Menschen eine Beziehung aufzubauen, erzählte ihm meinen Tagesablauf und stellte ihm Fragen, um selbst Entscheidungen zu treffen. [...] Wir waren ja immer zu zweit und ich wollte ihn nicht überrumpeln. Ich zweifelte, wie er entscheiden würde. Ich war ein Monster mit zwei denkenden Köpfen" (Gomez 2017).

Bei Johana Gomez beziehen sich die Fremdheitsgefühle auf ihr verändertes Körper-Selbst-Verhältnis, das durch die Gleichzeitigkeit der Vorstellung von Getrenntheit und Nichtgetrenntheit erheblich irritiert wird. Maggie Nelson (2017) dagegen bezieht ihr Befremden auf das Geschlecht des Kindes:

"Aus Gründen, die mir heute unerklärlich sind, weinte ich ein bisschen, als [...] der Ultraschalldiagnostiker uns [...] mitteilte, unser Baby sei ohne Zweifel ein Junge" (Nelson 2017, 113).

Während sie sich selbstreflexiv mit ihren Phantasien auseinandersetzt und vermutet, dass "die Trauer mit der Phantasie einer feministischen Tochter, einer Mini-Me" zusammenhinge, beschreibt Frau A. im Interview, das im Rahmen eines Forschungsprojekt zum Thema Mutterschaft geführt wurde, wie sie die Information erlebte, einen Jungen zu bekommen:

"Ich war irritiert von der Vorstellung, einen Penis im Bauch zu haben, ein Mädchen wäre mir einfach vertrauter. Ich bin ja auch weiblich und denke, dass ich mich besser einfühlen kann«.

In den beiden Sequenzen spielen Phantasien über das Geschlecht des Kindes eine wesentliche Rolle. Während Frau A. inzestuöse Phantasien mit dem als männlich identifizierten Kind abwehrt, indem sie die Vertrautheit körperlicher Gleichheit hervorhebt, führt gerade diese bei Nelson zu homosexuell gefärbten Phantasien. In beiden Beispielen sind diese leiblichen Empfindungen von hetero- und homosexuellen Vorstellungen geleitet, die sowohl biographisch als auch gesellschaftlich in die Körper 
eingeschrieben sind und das unbewusste Denken, Fühlen und Wahrnehmen mitbestimmen. Frau A identifiziert sich eindeutig heterosexuell und strukturiert ihre sexuellen Fantasien vor diesem Hintergrund heterosexuell. Bei Maggie Nelson können die sexuellen Fantasien ebenfalls auf ihre sexuelle Identität zurückgeführt werden. Sie lebt in einer queer-lesbischen Beziehung, in der die Partner ${ }^{\star}$ in zeitgleich mit Nelsons Schwangerschaft eine geschlechtliche Transformation durchlebt.

Der Einfluss der geschlechtlich-sexuellen Identitätsposition der Schwangeren kann in Verbindung mit dem Kinderwunsch auch als Überschreitung von Körpergrenzen phantasiert werden und eine schwangere Zwischenleiblichkeit erzeugen. Dies beschreibt ein Stundenprotokoll aus der Psychoanalyse von Frau Y:

Y.: "Ich habe geträumt, dass meine Tochter mit meinem Kind schwanger ist, und denke im Traum: Ich hab' die Arbeit und sie den tollen Bauch".

Im Traum inszeniert die Umkehrung der genealogischen Matrilinearität eine Beziehungskonstellation, in der die Tochter stellvertretend für die Mutter das Kind austrägt. Auch wenn Neid oder Wiedergutmachung narzisstischer Kränkungen diese Vorstellung beflügelt haben mögen, beeindruckt hier doch die phantasmatische Überschreitungen der Körperund Generationengrenzen, die der Kinderwunsch in Gang zu setzen vermag. Hier spannt sich ein phantasmatischer Zwischenraum zwischen Mutter und Tochter oder allgemeiner: zwischen zwei Frauen auf, in dem Schwanger-Sein als gemeinsame und als geteilte Erfahrung verortet wird. Diese Betrachtung dieses Erfahrungs- und Beziehungsraums wird im nächsten Abschnitt mit Blick auf Leihmutterschaft vertieft.

\section{Leihmutterschaft als leibliche Erfahrung und die Aufteilung des Mutterkörpers}

Da die Methoden der assistierten Reproduktion sowohl das Verständnis von Genealogie und Familie als auch neue Praxisformen von Mutterschaft ermöglicht haben, liegt es nahe zu fragen, wie sie auch die Erfahrungen von Schwangerschaft mitbestimmen. Dazu sollen im Folgenden Befunde einer ethnografischen Untersuchung von israelischen Leihmüttern (Teman 2010) ${ }^{2}$

2 Die Studie umfasst 43 Interviews mit 26 Leihmüttern und 45 Interviews mit 35 intended mothers zwischen 1998 und 2005, wobei 19 der Leihmütter und 23 der intended mothers nach der Geburt interviewt wurden. 
diskutiert werden. Wie wird Schwangerschaft angeeignet, wenn die körperlichen und sozialen Verflechtungen auf zwei Personen verteilt sind? Und was bedeutet dies für die bisherige Interpretation interpersonaler Verwobenheit zwischen Mutter ${ }^{\star}$ und Infans wie auch für die subjektiven Strategien, sich im Verlauf der Schwangerschaft das Fremde anzueignen?

Die von Emily Teman untersuchte Leihmutterschaft in Israel basiert auf einer staatlich reglementierten Vertragsbeziehung zwischen einem verheirateten heterosexuellen Paar und einer unverheirateten Frau, die Mutter mindestens eines Kindes ist. Auch der Kontakt zwischen Leihmutter und "intended mother/ parents « ${ }^{3}$ während des Schwangerschaftszeitraums und nach der Geburt ist durch staatliche Vorgaben geregelt. Teman charakterisiert die konkreten Schwangerschaftspraktiken der Leihmütter als aktive Aneignung der »tools of patriachy"; sie machen sich Medikalisierung, Objektivierung des weiblichen Körpers und Reproduktionstechnologien zunutze (Teman 2010, 53). Teman zeigt, dass Leihmütter den Dualismus von Natürlichkeit und Künstlichkeit in Bewegung bringen und zwischen drei Körpermodellen unterscheiden: "the natural everyday body, the natural pregnant body and the arificial surrogate body (ebd., 42). Die durch Leihmutterschaft bedingten körperlichen Veränderungen werden als "manifestations of the couple's nature " von der eigenen, davon unberührten persönlichen $\mathrm{Na}$ tur abgegrenzt (ebd., 51). Die Umgangsweisen der Leihmütter mit ihrem schwangeren Körper folgen verschiedenen Strategien: Mit dem Verweis auf die Unnatürlichkeit der Technologien findet eine Entkoppelung des Bauchs vom Rest der Person statt, indem der Bauch entpersonalisiert, sein Inhalt vom eigenen Körper abgetrennt und stattdessen mit der zukünftigen Mutter durch die Betonung der genetischen Abstammung verbunden wird. Diese phantasmatische Trennung geht mit einem spezifischen Umgang mit Emotionen einher: das Herz wird als prominenter Ort persönlicher Gefühle besetzt und vom sneutralisierten Bauch getrennt. Zudem werden Berührungen des eigenen schwangeren Bauches vermieden. Keine der Leihmütter berichtet von einer emotionalen Bindung zum Fötus während der Schwangerschaft und zum Kind nach der Geburt (ebd., 84).

Die Praktiken der Leihmütter können als bewusste Strategien der Körperfragmentierung angesehen werden. Während Körperfragmentierungen im klinisch-psychotherapeutischen Diskurs unbewusste Prozesse der Körperabspaltung infolge von traumatischen Erfahrungen repräsentieren,

3 Der Ausdruck intended mother beschreibt den Sachverhalt umfassender als eine deutsche Übersetzung, da auch der Prozess der emotionalen und sozialen Mutterwerdung mitbenannt wird. 
werden die Spaltungspraktiken hier aktiv als strategischer Schutzmechanismus vor ungewollter Bindung an das Infans und zur Vermeidung leiblich-affektiver Erfahrungen eingesetzt. Auf diese Weise werden die Leiberfahrungen in der Schwangerschaft abgewehrt und nicht - wie in den bisher vorgestellten empirischen Facetten - in einen leiblichen Zwischenraum integriert, der sich zwischen Mutter und Infans aufspannt. Während die Verwandtschaftsvorstellungen in den in Abschnitt II vorgestellten Zitaten durch eine leiblich-körperliche Koppelung mit dem Infans gekennzeichnet sind, akzentuieren die Leihmütter biologische Körpersubstanzen und genetische Verbindungen (Teman 2010, 111). Das Anliegen, ein genetisch eigenes Kind zu bekommen, bewirkt eine Fokussierung auf das Infans. Das externe Fühlen der Kindsbewegungen und die Wahrnehmung auf den Ultraschallbilder verstärkt bei der intended mother die Vorstellung, das Infans als eigenständiges, vom Mutterkörper getrenntes Wesen wahrzunehmen und eine emotionale Beziehung zu ihm herzustellen. Teman beschreibt, dass die Praktiken der intended mother den schwangeren Bauch der Leihmutter als Reproduktionsort besetzen und ihn sinto her own bodily schema as an appended extension " (ebd., 146) inkorporieren. Auf diese Weise entfaltet sich ein inter-uteriner Zwischenraum zwischen fruchtbarem und unfruchtbarem Uterus, in dem das Infans verortet wird und von beiden Mutterpositionen unterschiedlich emotional besetzt wird. Teman fasst diese Ausdehnung der Körpergrenzen mit dem Begriff des shifting body (ebd., 203).

Diese Art der Herstellung des phantasmatischen Körperraums zwischen beiden Mutterpositionen weist Parallelen zu der in Abschnitt II beschriebenen Traumsequenz auf. Während in dem Traumbeispiel transgenerationale Verwandtschaftsvorstellungen tragend sind, spielt bei den israelischen Leihmutterschaftsmöglichkeiten die soziale Anerkennung von Mutterschaft und weiblicher Identität eine zentrale Rolle. Die bevölkerungspolitische und nationale Bedeutung von Mutterschaft und der Institution Familie verstärkt die enge Verknüpfung von Mutterschaft mit Weiblichkeit. Dadurch wird eine psychische Gleichsetzung von Mutterschaft und Weiblichkeit begünstigt und ermöglicht beiden Mutterpositionen eine mütterliche Form der Körperbesetzung. Die Leihmutter erfährt über ihre natürliche Fruchtbarkeit einen Zugang zum sozialen mütterlichen Empowerment, die intended mother durch ihre Bereitschaft zu sozialer Mutterschaft.

Auch wenn die soziale Verankerung des Kinderwunsches für beide Mutterpositionen legitimatorische und narzisstische Bedeutungsebenen verbindet, werden hier sehr unterschiedliche Umgangsweisen in Bezug auf das 'Wunschkind deutlich, die auch mit Blick auf leibliche Mutter- 
schaft einen deutlichen Unterschied markieren. Neben der sozialen und psychischen Problematik schärfen die empirischen Einsichten in die psychischen Umgangsweisen der Leihmütter mit ihrem schwangeren Körper den Blick auf die körperbasierten Herausforderungen, den eigenen Körper für einen Anderen zur Verfügung zu stellen. Während das Fremde in der Schwangerschaft einer Mutter` mit eigenem Kind zwar psychisch sehr unterschiedlich (von angstvoll bis libidinös begehrt) angeeignet, aber in das eigene Körper-Selbst-Verhältnis integriert wird, unterstützt die Vorstellung des Fremden aus der Perspektive der Leihmutter die psychische Abwehr. Diese Abwehr- und Verschiebungsstrategien werden scheinbar weiträumig übertragen; Teman berichtet davon, dass die Schwangerschaft der Leihmutter von deren sozialem Umfeld häufig nicht wahrgenommen werde (Teman 2010, 203). Hier zeigen sich aufschlussreiche Parallelen zu Berichten über Körpererfahrungen von Frauen mit unerwünschten Schwangerschaften (Lundquist 2008). Auch zeugt es von einer besonderen Art von gesellschaftlicher Sprachlosigkeit, dass die reproduktionstechnischen Maßnahmen auf der körperlichen Ebene immer weiter spezifiziert werden, während die psychischen Herausforderungen und Folgen sowohl für Leihmütter wie auch für das Infans nahezu unerforscht sind.

Insgesamt illustrieren die Vignetten aus Abschnitt II und III die zentrale Funktion der Sprache als erkenntnisgenerierendes und anerkennendes Medium der Erfahrungen und rücken die Schwangeren als Subjekte von Erfahrung einschließlich ihrer somatischen und psychischen Integrationsleistungen in den Blick. Umgekehrt zeigen sie, dass die mangelnde Wahrnehmung und Anerkennung von Schwangerschaftserfahrungen durch vorgeformte Ansichten über das Mutter-Kind-Verhältnis Einsichten in subjektive Körpererfahrungen und sexuelle Intimverhältnisse verstellen.

\section{Fazit - Schwangeren eine Stimme geben}

Anhand verschiedener leiblicher und psychischer Erfahrungen mit dem körperlichen Prozess einer Schwangerschaft wurde gezeigt, dass neben einem Körperwissen auch die leibliche Erfahrung und die psychische Integration der Schwangerschaft von Bedeutung sind. Dies macht deutlich, dass Schwanger-Sein weit über den reproduktionsmedizinischen Diskurs eines somatischen Körperzustands hinausweist, aber auch mehr ist als eine weiblich-mütterliche Statuspassage (Mozygemba 2011). Schwangerschaft gestaltet sich, wie die empirischen Beispiele zeigen, als Beziehungsraum, in und an dem sich leiblich spürbare und phantasmatische Beziehungsvorstel- 
lungen mit dem unbekannten Anderen vollziehen. Dabei bezieht sich das Fremde als das Andere sowohl auf die unverfügbare somatische Präsenz des schwangeren Körpers als auch auf das Infans und Phantasien über den zukünftigen Mutterstatus. In diesem Zusammenhang vertieft ein Verständnis von Schwangerschaft als Zwischenleiblichkeit, dass die psychischen Aneignungs- und Abwehrprozesse durch ein Zusammenspiel von körperlichen, leiblichen und diskursiven Anrufungen hervorgebracht und beeinflusst werden. Die Unterschiedlichkeit der subjektiven Selbst- und Körper-Verhältnisse konkretisiert dabei nicht nur die subjektive Vielfalt psychischer Umgangsweisen, sondern dass und wie sozio-kulturelle Ideale und Anrufungen an Schwangerschaft als Orientierung für Umgangsweisen mit Schwangerschaft dienen. Dementsprechend spiegelt leibliches Spüren nicht nur eine performative Praxis wieder, sondern verweist auf eine aktive Position psychischer Verarbeitungsmöglichkeiten und subjektiver Selbstbestimmung. Dabei erweist sich die affektive Erfahrung des Leibes als Bedingung und Voraussetzung für einen selbstreflexiven psychischen Aneignungs- bzw. Abwehrprozess der Erfahrungen von Körperlichkeit in der Schwangerschaft und den damit verbundenen Subjektpositionen.

Gerade die Praxis der Leihmutterschaft zeigt in diesem Zusammenhang den Subjektstatus der Schwangeren im Umgang mit dem Erleben von intra-leiblichen Erfahrungen sehr deutlich. Zugleich werden darüber Fragen nach dem Verhältnis von Infans und schwangerer Mutter ${ }^{\star}$ sowie der Verweisungszusammenhang von Körperzustand der Schwangerschaft und Mutterschaft in einen erweiterten Zusammenhang gestellt. Damit werden nicht nur Annahmen eines authentischen und universellen Zugangs zu Schwangerschaftserfahrungen widerlegt, sondern gezeigt, dass der Prozess des Mutterwerdens primär eine psychische Leistung darstellt, der sich auch unabhängig von der leiblichen Erfahrung eines körperbasierten $\mathrm{Zu}$ stand einer Schwangerschaft vollziehen kann. Gerade an der phantasmatischen Annäherung der intended mothers an die Mutterposition sowie den unterschiedlich verwendeten Sprachbildern bezüglich der körperlichen und leiblichen Veränderungen (bspw. Zweck des Mutterseins, ein Monster mit zwei denkenden Köpfen) lässt sich der Bedeutungskontext des Gesellschaftlichen für die psychische Aneignung der Mutterposition verdeutlichen. Zudem veranschaulichen die Artikulationen der Schwangeren, dass Geschlechter- und Körperzuweisungen auf das somatische Erleben vorzugsweise dort greifen, wo leibliche Erfahrungen ignoriert und nicht in den psychischen Verarbeitungsprozess aufgenommen werden (können).

Eine Perspektive auf den Körper in der Schwangerschaft, die über eine medizinische Überwachung hinaus die erfahrungsbasierte Referenz leib- 
licher Empfindungen, Gefühle und Phantasien einbezieht, ist auch im Hinblick auf gesellschaftliche Anerkennungsverhältnisse von fundamentaler Bedeutung. Denn um subtile Formen der Körper-Selbst-Entfremdung in der Schwangerschaft in einer Matrix heteronormativer Deutungsmuster zu dekonstruieren und leibliche Erfahrungen für subjektive Selbstbestimmung produktiv zu machen, bedarf es (neuer) Begrifflichkeiten, um Schwangerschaft und Mutterschaft als Praktiken der Selbstbestimmung in der symbolischen Ordnung der Geschlechter zu etablieren.

\section{Literatur}

Abraham, Anke (2010): Körpertechnologien, das Soziale und der Mensch. In: Abraham, Anke/Müller, Beatrice (Hrsg.): Körperhandeln und Körpererleben. Multidisziplinäre Perspektiven auf ein brisantes Feld. Bielefeld, 113-138.

Achtelik, Kirsten (2015): Selbstbestimmte Norm. Feminismus, Pränataldiagnostik, Abtreibung. Berlin.

Auhagen-Stephanos, Ute (2017): Der Mutter-Embryo-Dialog. Fruchtbarkeit und Unfruchtbarkeit im Spiegel der Psychotherapie. Gießen.

Balsam, Rosemary (2012): Women's Bodies in Psychoanalysis. New York/London.

Duden, Barbara (2002): Die Gene im Kopf - der Fötus im Bauch. Historisches zum Frauenkörper. Hannover.

Duden, Barbara (1987): Geschichte unter der Haut. Ein Eisenacher Arzt und seine Patientinnen um 1730. Stuttgart.

Erikson, Susan L. (2012): Social Embodiments: Prenatal Risk in Postsocialist Germany. In: Anthropologica 54 [Nr. 1], 83-94.

Ettinger, Bracha (2006): The Matrixial Borderspace. Minneapolis.

Fonagy, Peter/Target, Mary (2003): Psychoanalyse und die Psychopathologie der Entwicklung. Stuttgart.

Gahlings, Ute (2006): Phänomenologie der weiblichen Leiberfahrungen. Freiburg, München.

Gomez, Johana (2017): Mutation of Body and Mind. https://www.adk-bw.de/gesturesmotherhood-gebaerden-der-mutterliebe.html (07.08.2018).

Heitzmann, Daniela (2017): Fortpflanzung und Geschlecht. Zur Konstruktion und Kategorisierung der generativen Praxis. Bielefeld.

Hirschhauer, Stefan/Heimerl, Birgit/Hoffman, Anika/Hofmann, Peter (2014): Soziologie der Schwangerschaft. Explorationen pränataler Sozialität. Stuttgart.

Jagenow, Angela/Mittag, Oskar (1984): Weiblicher Kinderwunsch und Sexualität. In: Psychosozial 21 [Nr. 7], 7-26.

Jäger, Ulle (2004): Der Körper, der Leib und die Soziologie. Entwurf einer Theorie der Inkorporierung. Königstein/Ts.

Kneuper, Elsbeth (2005): Mutterwerden in Deutschland: eine ethnologische Studie. (= Forum europäische Ethnologie 6). Münster. 
Kortendiek, Beate/Lange, Ute/Ullrich, Charlotte (2017): Schwangerschaft, Geburt und Säuglingszeit. Gender. Zeitschrift für Geschlecht, Kultur und Gesellschaft. Heft 2/2017.

Krüger-Kirn, Helga (2018): Care und Weiblichkeit. In: Freie Assoziation 1 »Familie und Geschlecht«, 68-73.

Krüger-Kirn, Helga (2017): Mutterschaft und Emanzipation revisited. Ein interdisziplinärer Dialog zwischen theoretischer Reflexion von Mutterschaft und körperlich-sinnlichem Erleben. In: Krüger-Kirn, Helga/Wolf, Laura (Hrsg.): Mutterschaft zwischen Konstruktion und Erfahrung. Berlin \& Toronto, 155-173.

Krüger-Kirn, Helga (2015a): Die konstruierte Frau und ihr Körper. Eine psychoanalytische, sozialwissenschaftliche und genderkritische Studie zu Körperpraktiken und Mutterschaft. Gießen.

Krüger-Kirn, Helga (2015b): Mutterschaft und weibliche Identität. In: Krüger-Kirn, Helga / Metz-Becker, Maritta / Rieken, Ingrid (Hrsg.): Mutterbilder. Kulturhistorische, sozialpolitische und psychoanalytische Perspektiven. Gießen, 125-147.

Lange, Ute/Ullrich, Charlotte (2018): Schwangerschaft und Geburt: Perspektiven und Studien aus der Geschlechterforschung. In: Kortendiek, Beate/Riegraf, Birgit/Sabisch, Katja (Hrsg.): Handbuch Interdisziplinäre Geschlechterforschung. Geschlecht und Gesellschaft, Wiesbaden, 1111-1119.

Lundquist, Caroline (2008): Being Torn. Toward a Phenomenology of Unwanted Pregnancy. In: Hypatia. A Journal of Feminist Philosophy 23 [Nr. 3], 136-155.

Malich, Lisa (2017): Die Gefühle der Schwangeren. Eine Geschichte somatischer Emotionalität (1780-2010). Bielefeld.

Makowsky, Katja / Schücking, Beate (2013): Was sagen die Mütter? Qualitative und quantitative Forschung rund um Schwangerschaft, Geburt und Wochenbett. Weinheim.

Mozygemba, Kati (2011): Die Schwangerschaft als Statuspassage. Das Einverleiben einer sozialen Rolle im Kontext einer nutzerorientierten Versorgung. Bern.

Nelson, Maggie (2017): Die Argonauten. Berlin.

Niekrenz, Yvonne (2016): Schwangerschaft und Geburt. In: Gugutzer, Robert/Klein, Gabriele / Meuser, Michael (Hrsg.): Handbuch Körpersoziologie. Band 2: Forschungsfelder und Methodische Zugänge. Wiesbaden, 217-229.

Sänger, Eva / Dörr, Annalena/Scheunemann, Judith/Treusch, Patricia (2013): Embodying Schwangerschaft: pränatales Eltern-Werden im Kontext medizinischer Risikodiskurse und Geschlechternormen. In: Gender. Zeitschrift für Geschlecht, Kultur und Gesellschaft 5 [Nr. 1], 56-71.

Schadler, Cornelia (2013): Vater, Mutter, Kind werden. Eine posthumanistische Ethnographie der Schwangerschaft. Bielefeld.

Schmitz, Hermann (1985): Phänomenologie der Leiblichkeit. In: Petzold, Hilarion (Hrsg.): Leiblichkeit. Philosophie, gesellschaftliche und therapeutische Perspektiven. Paderborn, 71-106.

Teman, Elly (2010): Birthing a mother. The Surrogate Body and the Pregnant Self. Oakland.

Tolasch, Eva / Seehaus, Rhea (2017): Mutterschaften sichtbar machen: Sozial- und kulturwissenschaftliche Beiträge. Berlin / Toronto.

Young, Iris Marion (2005): On Female Body Experience: "Throwing Like a Girl" and Other Essays. New York. 